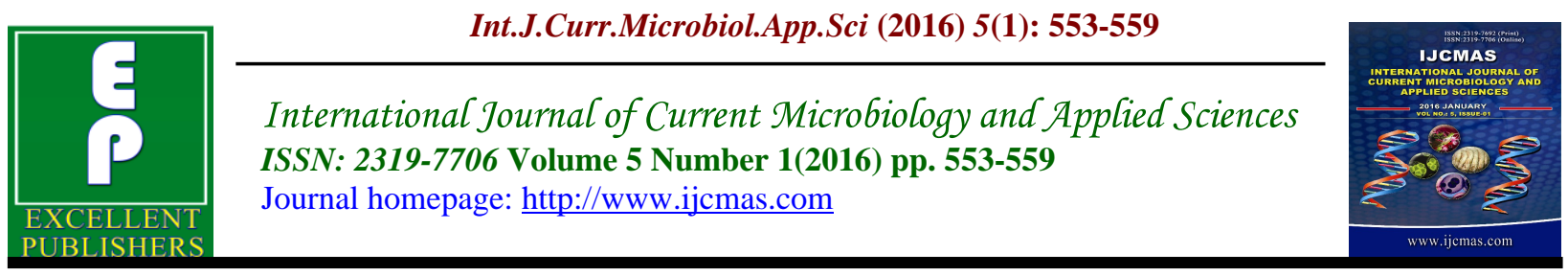

Original Research Article

hittp: ///dx.doi.org/10.20546/ijcmas.2016.501.056

\title{
Phenotypic Differentiation of Metallo beta Lactamases and Class A KPC Carbapenemases in Enterobacteriaceae Isolates
}

\author{
Gomty Mahajan*, Shashi Chopra, Jaspal Kaur, Sheevani and Kailash Chand \\ Department of Microbiology, Punjab institute of Medical Sciences, Jalandhar, India \\ *Corresponding author
}

\begin{abstract}
A B S T R A C T
Key words

Class A KPC,

Metallo beta

lactamases,

PBA,

EDTA,

Carbapenem

Resistant

Enterobacteriace

ae (CRE)

Article Info

Accepted:

25 December 2015

Available Online:

10 January 2016

Carbapenemase producers are increasingly reported worldwide in Enterobacteriaceae. Carbapenems are usually the choice of antimicrobials in infections caused by Enterobacteriaceae. Resistance to carbapenems is mostly due to production of enzymes - Carbapenemases, which are divided into Ambler Classes A, B and D. Phenotypic detection and differentiation of carbapenemases in Enterobacteriaceae is of great help to the patients as well as clinicians for proper infection control and appropriate patient management. The aim of the present study was to evaluate the performance of modified Hodge test and combined disc test for detection and differentiation of carbapenemase production. A study was conducted on 41 Carbapenem Resistant Enterobacteriaceae (CRE) strains which were isolated from various clinical samples over a period of one year (September 2013-Agust 2014). All the isolates were identified by standard microbiological procedures and antibiotic sensitivity was performed according to CLSI guidelines. Isolates were tested for carbapenemase production by modified Hodge test (MHT) and combined disc test. Phenotypic confirmatory test was done by using discs of Meropenem alone and those with phenyl boronic acid (PBA) or Ethylenediamnetetra acetic acid (EDTA) or both, for the detection of carbapenemase production and differentiation of KPC and MBL enzymes. Out of these 482strains, 41 (8.5\%) strains were resistant to carbapenems. Among the 41 carbapenem resistant isolates, 29 isolates were positive and 12 isolates were negative by MHT. Of these 41 carbapenem resistant clinical isolates of Enterobacteriaceae, 29 isolates were producing KPC, MBL and KPC+MBL enzymes. Rest were negative for KPC and MBL by combined disc test. We conclude that the need of the hour is simple, rapid and cost effective tests which will be able to identify and distinguish resistant and can be easily incorporated in routine microbiology lab testing.
\end{abstract}

\section{Introduction}

Carbapenems first introduced in 1980 are now frequently used as a reserved drug in treating serious infections caused by multidrug resistant gram negative bacilli.
Carbapenems are a group of $\beta$-lactam antimicrobial agents with an exceptionally broad spectrum of activity and often used as antibiotics of last resort for treating 
infections due to multidrug-resistant gramnegative bacilli since they are stable even in response to extended-spectrum and Amp C $\beta$-lactamases (Ikonomidis A et al, 2005). However, the emergence and proliferation of bacteria resistant to this important group of drug is jeopardizing the use of carbapenems. Resistance to carbapenem mostly is due to production of enzymes-Carbapenemases that hydrolyse carbapenems and other $\beta$-lactams. Carbapenemase enzymes fall into Ambler classification - A, B and D. These enzymes efficiently hydrolyse all beta-lactams except monobactams. They have shown a worldwide dissemination although they are more frequently reported in southern Europe and the Asiatic-Pacific region (Queenan AM et al, 2007).

More recently, a new type of class A betalactamase, the Klebsiella pneumoniae carbapenemase (KPC), has also spread among K. pneumoniae isolates and other Enterobacteriaceae. This enzyme confers various levels of resistance to all betalactams including carbapenems, even though cephamycins and ceftazidime are only weakly hydrolysed. Such co-existence of carbapenem-hydrolysing enzymes in enterobacterial pathogens may further compromise the therapeutic alternatives not only due to the carbapenemase mediated resistance to every beta-lactam, but also due to the linkage with non-beta-lactam resistance determinants. It also has several epidemiological implications, since MBLs and KPCs are mostly transposon- and/or integron-encoded determinants that can easily disseminate to other enterobacterial strains (Thomson KS et al, 2010). The Enterobacteriaceae isolates that harbour both MBL and KPC carbapenemases are increasingly recovered from clinical specimens and this has led to difficulty in differentiating and identifying these enzymes (Bansal et al., 2013). Appropriate detection of CRE is vital for patient care in order to institute correct therapeutic options. Molecular techniques can easily differentiate between these classes of carbapenemases but is available in only a few reference laboratories. Clinical and Laboratory Standards Institute incorporated the modified Hodge test (MHT) for the detection of carbapenemases (CLSI M100S21), but need of the hour is a rapid, practical, phenotypic method which can differentiate KPCs and MBLs in Enterobacteriaceae

\section{Materials and Methods}

\section{Bacterial Isolates}

A prospective study was conducted for a period of one year (October 2013September 2014) in the Microbiology lab, at a tertiary care hospital in northern India. All Enterobacteraceae isolates (Escherichia coli, Klebsiella species , Citrobacter species, Enterobacter species etc.) obtained from clinical samples like pus, wound swabs, body fluids, sputum, throat swab and endotracheal secretions were collected over a period of one year. The standard microbiological techniques were used for the isolation and identification of the isolates.

\section{Carbapenem Susceptibility}

Carbapenem Susceptibility of these isolates were tested for meropenem $(10 \mu \mathrm{g})(\mathrm{Hi}$ Media ) by disc diffusion method according to CLSI criteria (CLSI 2013) . Of these isolates, strains which were resistant to meropenem (30 mcg) on routine disc diffusion testing were included in the study. These resistant strains were screened for carbapenemase production by using Modified Hodge test. A combined disc test was performed as a confirmatory phenotypic 
method, to differentiate between MBL and KPC productions.

\section{Procedure for Modified Hodge Test}

All CRE isolates detected by disc diffusion were also tested by CLSI recommended Modified Hodge test. A Mueller Hinton Agar plate was inoculated with a $0.5 \mathrm{Mc}$ Farland's suspension of E. coli, ATCC 25922 and it was streaked to obtain confluent growth, by using a swab. A $10 \mu \mathrm{g}$ meropenem disk was placed at its centre, and each isolate was streaked from the disk to the edge of the plate and plate was incubated at $37^{\circ} \mathrm{C}$ overnight. After incubation, the plates were examined for a clover leaf type of indentation at the intersection of growth of the test organism and the E. coli ATCC 25922, within the zone of inhibition of the carbapenem susceptibility disc. (CLSI,2011)

A positive test shows a clover leaf like indentation of E. coli ATCC 25922 which grows along the growth of test organism within the disc diffusion zone. A negative test shows no growth of E. coli ATCC 25922 along the growth of test organism within the disc diffusion zone.(CLSI,2011)

\section{Procedure for Confirmatory Phenotypic Test}

Use of inhibitor phenylboronic acid (PBA), EDTA or both along with meropenem disc was used for detection of KPC and MBL, respectively. A phenotypic detection test was done by using combined discs of meropenem alone and with those of phenyl boronic acid (PBA) or EDTA or both PBA and EDTA, for the detection of carbapenemase production and differentiation of KPC and MBL enzymes. The stock solution of PBA was prepared by dissolving phenyl boronic acid (Sigma-
Aldrich, Germany ) in DMSO at a concentration of $20 \mu \mathrm{g} / \mathrm{ml}$. $20 \mu \mathrm{L}$ of the stock solution (containing $400 \mu \mathrm{g}$ of PBA) was dispensed onto commercially available meropenem discs (Hi-media). The stock solution of EDTA (Sigma-Aldrich, Germany) was prepared by dissolving anhydrous EDTA in distilled water at a concentration of $0.1 \mathrm{M}$. From this solution, $10 \mu \mathrm{L}$ (containing $292 \mu \mathrm{g}$ of EDTA) was dispensed onto Meropenem discs. The discs were dried and used within 60 minutes. The test was performed by inoculating the test organism on Mueller Hinton Agar and placing one disc of Meropenem without any inhibitor and three discs of Meropenem, each containing containing $400 \mu \mathrm{g}$ of PBA

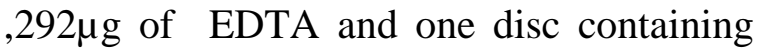
both i.e. $400 \mu \mathrm{g}$ of PBA and $292 \mu \mathrm{g}$ of EDTA on it. The agar plates were incubated at $37^{\circ} \mathrm{C}$ overnight. The diameter of the growth inhibitory zone seen around the Meropenem disc with PBA, EDTA, PBA+EDTA was compared with that seen around the plain Meropenem disc.(Tsakris A et al, 2010).

Production of KPC was considered when the growth inhibitory zone diameters seen around the Meropenem disc with PBA and the Meropenem disc with PBA+EDTA has increased to $>=5 \mathrm{~mm}$ as compared to the growth inhibitory zone diameter seen around the disc containing Meropenem alone. Production of MBL was considered when the growth inhibitory zone diameters seen around the Meropenem disc with EDTA and the Meropenem disc with PBA+EDTA had increased to $>=5 \mathrm{~mm}$ as compared to the growth inhibitory zone diameter seen around the disc containing Meropenem alone. Productions of both KPC and MBL were considered when the growth inhibitory zone diameter seen around the Meropenem disc with both PBA+EDTA had increased to $>=5 \mathrm{~mm}$, as compared to the growth inhibitory zone diameter seen around the 
disc containing Meropenem alone. When none of the three combined disc tests was positive, the isolate was considered to be negative for MBL and KPC carbapenemase productions. ( Tsakris A et al ,2010)

\section{Results and Discussion}

Total of $41(8.5 \%)$ carbapenem resistant Enterobacteraceae strains were isolated from 482 clinical samples. These included 18 strains of E. coli, 13 strains of Klebsiella spp., 04 strains of Citrobacter spp., 04 strains of Enterobacter spp. \& 2 strains of Proteus vulgaris. [Table1]. A majority $(28 \%)$ of the carbapenem resistant Enterobacteraceae isolates were isolated from endotracheal secretions. All CRE isolates were $100 \%$ sensitive to colistin, polymyxin B and tigecycline

These $41 \mathrm{CRE}$ isolates were subjected to screening for carbapenemase by Modified Hodge test. Out of these, 32 strains (78\%) showed positive results on doing Modified Hodge test. On the basis of combined discs test, 17 strains $(41.46 \%)$ were found to be MBL producers i.e. the growth inhibitory zone diameter around the meropenem disc with EDTA and the meropenem disc with PBA+EDTA had increased to $>=5 \mathrm{~mm}$ as compared to the growth inhibitory zone diameter seen around the disc containing meropenem alone.

Two (4.9\%) isolates were found to coproduce both MBL and KPC, as the growth inhibitory zone diameters seen around the Meropenem disc with both PBA+EDTA had increased to $>=5 \mathrm{~mm}$ as compared to the growth inhibitory zone diameter seen around the disc containing Meropenem alone. Ten isolates (24.4\%) were KPC producers. Twelve isolates were considered negative for MBL and KPC production, as none of the three combineddisc tests was positive [Table 1].
Currently MBLs and KPCs are considered a major threat in Enterobacteriaceae, representing a potential source of clinical failure in patients treated with almost all beta lactam regimens (Nordmann $\mathrm{P}$ et al, 2009). The emergence of carbapenemhydrolyzing beta-lactamases has threatened the clinical utility of this antibiotic class and it has brought us closer to the challenge of 'Pan Drug Resistance' in Gram-negative bacteria. The prevalence of Carbapenemase producing Enterobacteriaceae varies from place to place and with geographical regions. The prevalence of Carbapenemase Producing Enterobacteriaceae reported from India range from 7 to $51 \%$. ( Datta $\mathrm{P}$ et al, 2012); (Wattal C et al, 2010). We have reported $8.5 \%$ of CRE prevalence in our Institute. This resistance to carbapenem is low as compared to studies by various authors from India. Wattal $\mathrm{C}$ et al reported high prevalence of resistance to carbapenems ranging from 13 to $51 \%$ in $\mathrm{E}$. coli and Klebsiella spp. from ICUs and wards from a tertiary care hospital in Delhi. Few authors have reported high prevalence of resistance varying from 17 to $22 \%$ to various carbapenems among Enterobacteriaceae strains. (Gupta et al,2006) $100 \%$ sensitivities to colistin, polymyxin B and tigecycline were noted for these CRE isolates. Our findings are in sync with those reported in literature and have stated that tigecycline and polymyxins remained the most active in-vitro drug for the CRE isolates.

In our study out of 41Carbapenemase producing Enterobacteriaceae isolates, $24.4 \%$ were KPC producers, $41.46 \%$ were MBL producers and $4.9 \%$ were both KPC and MBL producers. Results of a study conducted by Baraniak, $\mathrm{J}$ et al were comparable to ours as they reported $57.9 \%$ KPC producers and, and $43.4 \% \mathrm{MBL}$ producers (Barniak $\mathbf{J}$ et al,2013). Tsakris A et al reported out of 141 carbapenemase- 
positive Enterobacteriaceae isolates, $44.68 \%$ were KPC producers, $33.33 \%$ were MBL producers, and $21.98 \%$ were KPC and
MBL producers. They reported Triple Disc Test $100 \%$ sensitive for detection of KPC \& MBL (Tsakris et al 2010).

Table.1 Detection of Carbapenemase Production by Modified Hodge Test and Combined Disc Test among the Clinical Isolates of Enterobacteriaceae

\begin{tabular}{|c|c|c|c|c|c|c|c|}
\hline Organism & $\begin{array}{l}\text { No. of } \\
\text { strains } \\
\text { isolated }\end{array}$ & KPC & $\%$ & $M B L$ & $\%$ & Both & $\%$ \\
\hline E.coli & 18 & 5 & 27.8 & 7 & 38.9 & 1 & 5.6 \\
\hline Klebsiella spp. & 13 & 3 & 23 & 5 & 38.5 & 1 & 7.7 \\
\hline $\begin{array}{l}\text { Citrobacter } \\
\text { spp. }\end{array}$ & 04 & 1 & 25 & 2 & 50 & 0 & 0 \\
\hline $\begin{array}{l}\text { Enterobacter } \\
\text { spp. }\end{array}$ & 04 & 1 & 25 & 2 & 50 & 0 & 0 \\
\hline Proteus spp & 02 & 0 & 0 & 1 & 50 & 0 & 0 \\
\hline Total & 41 & 10 & 24.4 & 17 & 41.46 & 2 & 4.9 \\
\hline
\end{tabular}

In the present study, 32 strains out of 41 CRE strains showed modified Hodge test (MHT) positive. Among the 50 carbapenem resistant isolates, only $17(34 \%)$ isolates were positive and $33(66 \%)$ isolates were negative by MHT. In contrast to the present study Deshpande et al. reported $91.6 \%$ were MHT positive.( Deshpande et al. 2010) Furthermore, high false-positive rates have been reported. These are mainly caused by CTX-M type ESBLs and to a lesser extent, AmpC hyperproduction and ESBL production coupled with porin loss or porin mutations (Pasteran et al., 2010; Carvalhaes et al, 2010). Apart from being technically demanding and time consuming, disadvantages of this test (MHT) include possible interpretation difficulties and the inability to distinguish between different classes of carbapenemases (Tsakris et al., 2009; Hirsch and Tam, 2010). Twelve strains were resistant to carbapenem but both MBL and KPC production test were negative in these isolates. Since another important cause of carbapenem resistance among Enterobacteriaceae could be overproduction of ESBL or Amp C enzyme with porin loss, this could be the reason of resistance in these few bacterial strains.

Enterobacteriaceae majorly contribute to the intrinsic human gut flora. They are also capable of colonizing the gut of patients and spreading through the community via the faeco-oral route. Hence the spread of carbapenemase resistant Enterobacteriaceae is deeply alarming in a country such as India with a reservoir of more than 1.4 billion people (Nordman et al., 2011; Paterson, 2006).

Additionally detection and surveillance of CRE has become a matter of major importance for the selection of appropriate therapeutic schemes and implementation of infection control measures (Miriagou et al, 2010) One drawback of our study is the inability to compare the phenotypic methods with PCR for detection for CRE. Molecular methods like PCR, DNA hybridization and sequencing are the gold standard for detection of carbapenemase production. But 
these are used in research settings and not in routine diagnostic laboratories. The need of the hour is simple, rapid and cost effective tests which will be able to identify and distinguish resistant pathogens for improved patient outcome, facilitating efficient infection control and reducing the escalation of resistance (Thomson KS , 2010).

We conclude that Triple Disc Test for KPC and MBL production is simple to perform and materials used are cost-effective, non toxic and easily available which makes it highly pertinent as screening test in routine clinical laboratory. With this it also guides the clinician regarding empirical treatment.

\section{References}

Bansal M, Vyas N, Sharma, .Maheshwari RK. (2013). Indian Journal of Basic and Applied Medical Research;Vol.-3, Issue-1, P.314-320

Baraniak, J. Fiett, M. Herda, E. Nikonorow, N. Skrzypczynska, W. Hryniewicz, L.P.G. Derde, M.J.M. Bonten et al.(2013) Species distribution, clonal structure and carbapenemase types of carbapenemase-producing

Enterobacteriaceae (CPE) in European and Israeli hospitals:a comparative study.

Carvalhaes, C.G.( 2010). Cloverleaf test (modified Hodge test) for detecting carbapenemase production in Klebsiella pneumoniae: be aware of false positive results. J. Antimicrob. Chemother., 65: 249-251.

Clinical and Laboratory Standards Institute. Performance Standards for Antimicrobial Susceptibility Testing: Twenty First Informational Supplement M100-S21. CLSI, Wayne, PA: USA; 2011.

Datta P, Gupta V, Garg S, Chander J (2012). Phenotypic method for differentiation of carbapenemases in Enterobacteriaceae: Study from north India. Indian $\mathrm{J}$ Pathol Microbiol; 55:357-60 13.

Deshpande, P., Rodrigues, C., Shetty, A., et al. (2010). New Delhi Metallobetalactamase (NDM-1) in Enterobacteriaceae: Treatment options with carbapenems compromised. J. Assoc. Physicians India, 58: 147-9.

Gupta E, Mohanty S, Sood S, Dhawan B, Das BK, Kapil A. (2006). Emerging resistance to carbapenems in a tertiary care hospital in North India. Indian $\mathbf{J}$ Med Res, 124:95-8

Hirsch, E.B., Tam, V.H. (2010). Detection and treatment options for Klebsiella pneumonia carbapenemases (KPCs): an emerging cause of multidrugresistant infection. J. Antimicrob. Chemother., 48: 1323-1332.

Ikonomidis A, Tokatlidou D, Kristo I . (2005). Outbreaks in distinct regions due to a single Klebsiella pneumoniae clone carrying a blaVIM-1 metallo-blactamase gene. J Clin Microbiol; 43: 5344-7.

Miriagou V, Cornaglia G, Edelstein M, Galani I, Giske CG, Gniadkowski M, et al. (2010). Acquired carbapenemases in Gram-negative bacterial pathogens: detection and surveillance issues. Clin Microbiol Infect;16:112-22.

Nordman, P., Nass, T., Poirel, L.(2011). Global spread of carbapenemase producing Enterobacteriaceae. Emerg. Infect. Dis., 17: 1791-1798.

Pasteran F, Mendez T, Guerriero L et al.(2009) Sensitive screening tests for suspected class A carbapenemase production in species of Enterobacteriaceae. J Clin Microbiol ; 47: 1631-9. 
Paterson D.L. (2006). Resistance in gram negative bacteria: Enterobacteriaceae. Am. J. Infect. Control, 34: S64-S73.

Queenan AM, Bush K. Carbapenemases (2007). The versatile b-lactamases. Clin Microbiol Rev; 20: 440-58

Thomson KS. Extended-SpectrumLactamase, ampC and carbapenemase issues. (2010). J $\quad$ Clin Microbiol;48:1019-25.

Tsakris A, Poulou A, Pournaras S, Voulgari E, Vrioni G, Themeli Digalaki K.(2010). A simple phenotypic method for the differentiation of metallo-lactamases and class A KPC carbapenemases in Enterobacteriaceae clinical isolates. J Antimicrob
Chemother;65:1664-71.( J Antimicrob Chemother 2010; 65: 1664-1671 doi:10.1093/jac/dkq210 Advance Access publication 11 June 2010)

Tsakris A, Poulou A, Themeli-Digalaki K et al. (2009). Use of boronic acid disk tests to detect extended-spectrum blactamases in clinical isolates of KPC carbapenemase-possessing Enterobacteriaceae. J Clin Microbiol; 47: 3420-6.

Wattal C, Goel N, Oberoi JK, Raveendran R, Datta S, Prasad KJ. (2010).Surveillance of multidrug resistant organisms in a tertiary care hospital in Delhi, India. J Assoc Physicians India;58:32-6.

\section{How to cite this article:}

Gomty Mahajan, Shashi Chopra, Jaspal Kaur, Sheevani and Kailash Chand. 2016. Phenotypic Differentiation of Metallo beta Lactamases and Class A KPC Carbapenemases in Enterobacteriaceae Isolates. Int.J.Curr.Microbiol.App.Sci. 5(1): 553-559

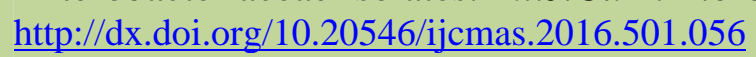

